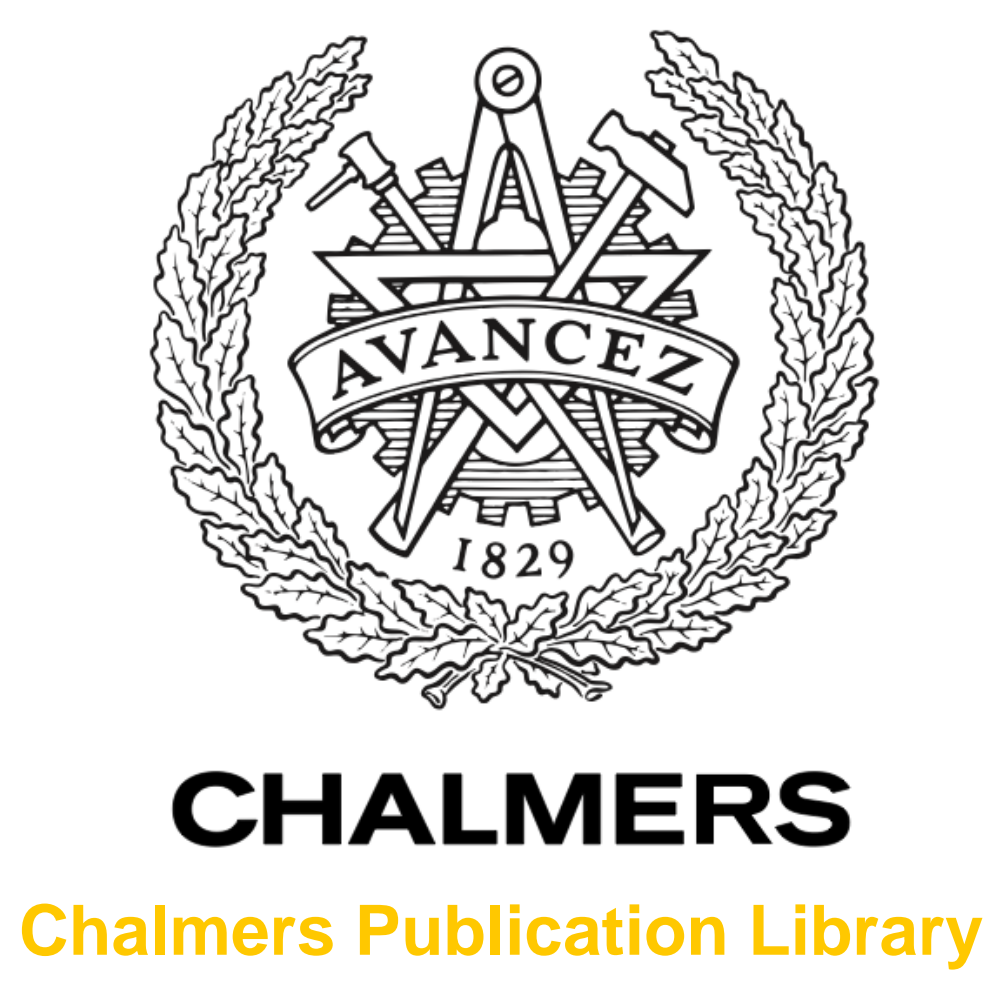

\title{
Performance study of fixed and moving relays for vehicular users with multi-cell handover under co-channel interference
}

This document has been downloaded from Chalmers Publication Library (CPL). It is the author's version of a work that was accepted for publication in:

\section{IEEE International Conference on Connected Vehicles and Expo}

Citation for the published paper:

Yutao, S. ; Ren, Z. ; Wanlu, S. (2013) "Performance study of fixed and moving relays for vehicular users with multi-cell handover under co-channel interference". IEEE International Conference on Connected Vehicles and Expo

Downloaded from: http://publications.lib.chalmers.se/publication/191327

Notice: Changes introduced as a result of publishing processes such as copy-editing and formatting may not be reflected in this document. For a definitive version of this work, please refer to the published source. Please note that access to the published version might require a subscription. 


\title{
Performance Study of Fixed and Moving Relays for Vehicular Users with Multi-cell Handover under Co-channel Interference
}

\author{
Yutao Sui, Zhe Ren, Wanlu Sun, Tommy Svensson, and Peter Fertl
}

\begin{abstract}
In this paper, we investigate the power outage probability (OP) of a vehicular user equipment (VUE) device served by half-duplex decode-and-forward relay nodes (RNs) under cochannel interference. Both moving RNs (MRNs) and fixed RNs (FRNs) are studied, and compared with the baseline, base station (BS) to VUE direct transmission. In order to understand the benefit for vehicular users served by an $\mathrm{RN}$, we consider practical channel models for different involved links as well as the impact of handover (HO) between the BS and the RNs. For an accurate comparison, we present a comprehensive framework to optimize the HO parameters, as well as we numerically optimize the FRN position which minimizes the average power OP at the VUE. FRN shows its advantage to serve its nearby VUEs. However, when vehicular penetration loss is moderate to high, MRN assisted transmission greatly outperforms transmission assisted by an FRN as well as direct transmission. Hence, the use of MRNs is very promising for improving the quality-of-service $(\mathrm{QoS})$ of VUEs in future mobile communication systems.
\end{abstract}

\section{INTRODUCTION}

In the near future, public transportation vehicles, e.g., buses, trams, or trains, will become natural hotspots for wireless data traffics, due to the high market penetration of smartphones, tablets and the increasing portability of laptops. Beyond the year of 2020, as predicted by the European Union project Mobile and wireless communications Enablers for the Twentytwenty Information Society (METIS), it will be fairly common to have up to 50 active vehicular user equipment (VUE) devices per bus and up to 300 active VUE devices per train [1]. Thus, how to improve the quality-of-service (QoS) at the VUEs is an important task in the next generation mobile communication systems.

The use of moving relay nodes (MRNs) in cellular systems was investigated in the 3rd Generation Partnership Project (3GPP) Long Term Evolution (LTE) system as an effective way to serve VUEs that are affected by moderate to high vehicular penetration loss (VPL) [2]. Measurements show that VPL can be as high as $25 \mathrm{~dB}$ in a minivan at the frequency of $2.4 \mathrm{GHz}$ [3], and higher VPLs are foreseeable in the well isolated vehicles of our interest in higher frequency bands, e.g., the $3.6 \mathrm{GHz}$ frequency band allocated to next generation mobile communication at the World Radio Communication Conference in 2007. One of the major advantages of using MRNs is to eliminate the effect of VPL, which significantly reduces the outdoor to indoor signal strength. By using two separate indoor and outdoor antennas connected through a

Yutao Sui, Wanlu Sun, and Tommy Svensson are with the Department of Signals and Systems, Chalmers University of Technology, Gothenburg, Sweden, Email:\{sui.yutao, wanlu, tommy.svensson\}@chalmers.se. Zhe Ren and Peter Fertl are with the BMW Group Research and Technology, Hanauser Strasse 46, Munich, Germany, Email: \{zhe.ren, peter.fertl\}@bmw.de. cable introducing negligible losses, MRNs can circumvent the VPL, and thereby improve the received signal strength at the VUE. Furthermore, since an MRN can create its own cell within a vehicle, group handover (HO) of VUEs served by the same MRN can be performed, which could lower the HO failure probabilities of the VUEs [4]. Thus, MRNs are potentially beneficial to serve VUEs.

Studies have shown that by deploying coordinated and cooperative relays on top of trains, the QoS of a VUE inside the vehicle can be significantly improved [5]. In [6], it is shown that in a noise limited system, using MRNs can improve the spectral efficiency and reduce the power outage probability (OP) for VUEs when the average transmit power of the BS and the relay node (RN) is fixed. In [7], the performance of using MRN where the communication is corrupted by co-channel interference (CCI) is studied. However, the benefits of using MRNs still need to be justified in more practical setups.

In this paper, we aim at evaluating the OP performance at a VUE by modeling the effects of pathloss, shadowing, small scale fading and CCI between different nodes according to practical propagation conditions, as well as considering the impact of $\mathrm{HO}$ between different nodes. Moreover, we investigate a general scenario that considers deploying MRNs on top of public transportation vehicles, and compare the OP performance of a VUE under dual-hop FRN and MRN assisted transmission with the baseline direct single-hop BS-to-VUE transmission. To facilitate our comparisons, we also present a comprehensive discussion about how to optimize the $\mathrm{HO}$ parameters, as well as how to optimally deploy the FRN in order to minimize the average end-to-end OP of the VUE. It is shown that as the VPL increases, an MRN is better at lowering the OP of VUEs than the BS-to-VUE direct transmission as well as the FRN assisted scheme.

\section{SYSTEM MODEL}

We consider the downlink of an RN assisted system with two cells: one primary cell where the OP performance at the VUE is investigated and one interfering cell (see Fig. 1). For convenience, we label all the nodes in the primary cell with number 1 and nodes in the interfering cell with number 2 . We assume that all transmitters, i.e., BSs, FRNs and MRNs, are transmitting at fixed average power and no power control schemes are considered. The BSs in both cells have a fixed coverage of $D$ meters and vehicles move along a highway. A symmetric deployment of FRNs is considered, i.e., each of the FRNs, both in the primary and the interfering cell, is at the same distance of $d$ meters from its serving BS. For MRN assisted transmission, we assume that the MRN is deployed 


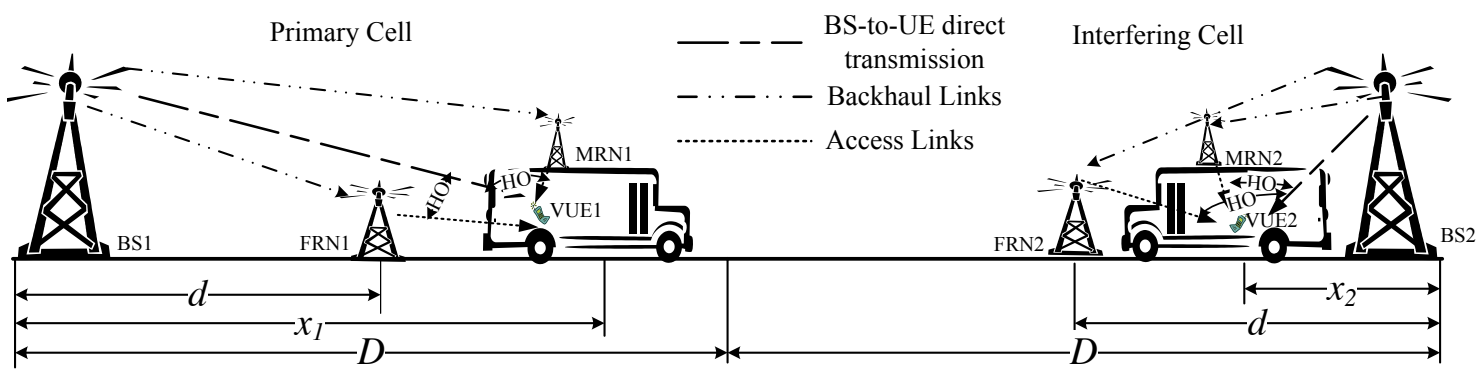

Figure 1. System model, the OP performance at the VUE is investigated in the primary cell while CCI is coming from the interfering cell. HO is enabled between the BS and the FRN or the MRN, i.e., FRNs or MRNs are constituting own cells.

on top of a vehicle, and it eliminates the VPL by properly separating its indoor and outdoor antennas. The BS-RN and RN-UE links are denoted as backhaul link and access link, respectively. It is assumed that both the MRN and the FRN are decode-and-forward (DF) and half-duplex, i.e., in the first hop the BS transmits to the $\mathrm{RN}$ and the $\mathrm{RN}$ decodes the received signal, while in the second hop, the RN forwards the decoded symbol to the VUE. The same types of RNs are assumed to be used in the two cells, i.e., scenarios such as one cell being equipped with MRN while the other uses FRN is not considered in this work. We assume the VUE can be handed over between the BS and the FRN or the MRN, and the $\mathrm{HO}$ decisions of the primary and interfering cell are made independently. Therefore, the interference experienced by the MRN backhaul and the VUE in the primary cell depends on the transmission mode of the interfering cell.

In general, when considering only a single interference source, the received signal-to-interference-plus-noise ratio (SINR) at a receiver (RX) can be expressed as

$$
\gamma=\frac{P_{r}(y)}{P_{r_{\mathrm{I}}}\left(y_{\mathrm{I}}\right)+N_{0}},
$$

where $P_{r}(y)$ denotes the received desired signal power and $P_{r_{\mathrm{I}}}\left(y_{\mathrm{I}}\right)$ is interference power at the RX; $y$ is the distance between the transmitter (TX) and the (RX) and $y_{\mathrm{I}}$ represents the distance between the interference source and the RX; $N_{0}$ is the average background noise power. If the TX has an average transmit power $P_{\mathrm{t}}$ and the $\mathrm{RX}$ is at a distance of $y$ from the $\mathrm{TX}$, the received desired signal power $P_{r}(y)$ at an RX is given as

$$
P_{r}(y)=P_{\mathrm{t}} L(y) \psi|h|^{2},
$$

where $L(y)$ models the pathloss when an $\mathrm{RX}$ is at distance $y$ from the TX. Moreover, $\psi$ denotes the power loss caused by shadowing and $h$ represents the respective small scale fading coefficient. Without loss of generality, we consider a flat fading environment. In wideband systems employing orthogonal frequency-division multiple access (OFDMA), this setup can be seen as a subchannel or a subchannel group whose bandwidth is much smaller than the coherence bandwidth of the channel [8, Ch. 12]. Similarly arguments hold for the interference links. The propagation models applied in this study will be detailed in Section V.

As both FRNs and MRNs are deployed outdoors, the VPL does not affect the their backhaul connections. The received SINR at the RNs can be directly obtained from (1). However, the BS1 to UE1 direct transmission and the access links of the FRN assisted schemes are impaired by the VPL. We assume that both the vehicles in the primary and interfering cells have the same VPL of value $\varepsilon$, where $0<\varepsilon \leq 1$. Thus, for the direct transmission from BS1 to UE1, the received SINR is $\gamma_{\mathrm{D}}=\frac{P_{r_{\mathrm{D}}}\left(y_{\mathrm{D}}\right) \varepsilon}{P_{r_{\mathrm{I}_{\mathrm{D}}}}\left(y_{\mathrm{I}_{\mathrm{D}}}\right) \varepsilon+N_{0}}$, and for the access link of the FRN assisted transmission we have $\gamma_{\mathrm{Fa}}=\frac{P_{r_{\mathrm{Fa}}}\left(y_{\mathrm{Fa}}\right) \varepsilon}{P_{r_{\mathrm{I}_{\mathrm{Fa}}}}\left(y_{\mathrm{I}} y_{\mathrm{Fa}}\right) \varepsilon+N_{0}}$. For the MRN assisted transmission, as we assumed the antenna communicating with the VUE is deployed inside the vehicle, there is no VPL affecting the desired signal; however, the interfering power of the MRN access link, i.e., MRN2UE1, is attenuated twice by both vehicles. Thus, we have $\gamma_{\mathrm{Ma}}=\frac{P_{r_{\mathrm{Ma}}}\left(y_{\mathrm{Ma}}\right)}{P_{r_{\mathrm{M}}}\left(y_{\mathrm{I}_{\mathrm{Ma}}}\right) \varepsilon^{2}+N_{0}}$.

\section{Outage Analysis}

In the presence of fading, there is always a probability that the received SINR at the receiver is below a given threshold to support a required transmission rate of $R$ bits/sec/Hz. In addition, from a QoS point of view, all kinds of services have some minimum bit error rate or codeword error rate requirements, which can be translated to a required average minimum received SINR at the receiver [8, Ch 12]. The probability that the received SINR $\gamma$ falls below a given threshold $\gamma_{\text {th }}$ is usually referred to as OP. For direct transmission, the OP at the VUE is calculated as

$$
P_{\text {out }_{\mathrm{D}}}\left(\gamma_{\mathrm{th}_{\mathrm{D}}}\right)=\operatorname{Pr}\left(\gamma<\gamma_{\mathrm{th}_{\mathrm{D}}}\right) .
$$

For the MRN assisted transmission, as we assume no fading for the access link, the OP expression is similar to (3), but with a different threshold $\gamma_{\mathrm{th}_{\mathrm{R}}}$ due to the half-duplex loss, which will be detailed in a later part of this section.

In a half-duplex DF FRN-assisted system, however, an outage happens if either the backhaul or the access link is in outage. Hence, for FRN assisted transmission, the OP is given as

$$
P_{\text {out }_{\mathrm{R}}}\left(\gamma_{\mathrm{th}_{\mathrm{R}}}\right)=\operatorname{Pr}\left(\min \left(\gamma_{\mathrm{bk}}, \gamma_{\mathrm{ac}}\right)<\gamma_{\mathrm{th}_{\mathrm{R}}}\right) .
$$

When only considering the effect of small scale fading, the closed form solution of OP were derived in [9] and the corresponding OP performance of FRN and MRN with various VPL was studied in [7]. However, the exact OP expression when taking both shadowing and small scale fading into account in the presence of CCI with non-negligible background noise, is still unknown. Thus, in this study, we resort to system level 
evaluations to compare the OP performance among different schemes.

The threshold $\gamma_{\mathrm{th}_{\mathrm{D}}}$ or $\gamma_{\mathrm{th}_{\mathrm{R}}}$ varies according to different QoS requirements, and in this study, we choose the thresholds based on the achievable rate in an LTE system studied in [10] as

$$
R(\mathrm{bits} / \mathrm{sec} / \mathrm{Hz})=B_{\mathrm{ef}} \eta \log _{2}\left(1+\frac{\gamma}{\gamma_{\mathrm{ef}}}\right),
$$

where $B_{\text {ef }}$ adjusts for the bandwidth efficiency, $\gamma_{\text {ef }}$ amends the SINR implementation efficiency of the system, and $\eta$ is a correction factor. In a single antenna LTE setup with fast time and frequency domain packet scheduling, $B_{\text {ef }}$ and $\gamma_{\text {ef }}$ are both found to be 0.62 , and $\eta$ is set to 1 [10].

From (5), we can obtain $\gamma_{\mathrm{th}_{\mathrm{D}}}=\gamma_{\mathrm{ef}}\left(2^{\frac{R}{B_{\mathrm{ef}} \eta}}-1\right)$ for direction transmission with a required end-to-end rate of $R$ bits/sec/Hz. However, a rate of $2 R$ bits $/ \mathrm{sec} / \mathrm{Hz}$ is required for both the backhaul and the access links in order to achieve an end-to-end rate of $R$ bits/sec/Hz. Thereby, the threshold is set to be $\gamma_{\mathrm{th}_{\mathrm{R}}}=\gamma_{\mathrm{ef}}\left(2^{\frac{2 R}{B_{\mathrm{ef}} \eta}}-1\right)$.

\section{HANDOVER PARAMETER OPTIMIZATION AND OPTIMIZING THE POSITION OF FRNS}

\section{A. The considered $\mathrm{HO}$ mechanism}

$\mathrm{HO}$ is an indispensable part in a mobile communication system to support user mobility, and to achieve load balancing between different nodes. There are various events that can initialize $\mathrm{HO}$ procedures in a mobile communication system [11, Ch. 3, 4, 22 and 25]. In this work, we study the OP performance at the VUE by taking into account the impact of $\mathrm{HO}$ between the BS and the FRN or the MRN. We accommodate the A3 event defined in 3GPP LTE to trigger HO procedures, which is defined as: "Neighbor cell becomes better than an offset relative to the serving cell" [11, Ch. 3]. Sometimes, the offset in the A3 event is also referred to as $\mathrm{HO}$ hysteresis margin. In addition, to avoid the ping-pong effect, i.e., a user equipment (UE) is being handed forth and back between two neighboring nodes, the A3 event needs to be observed for a certain period of time, which is referred to as time-to-trigger (TTT), before performing the HO procedures. In practical systems, the signal strength is measured by using the reference signal received power (RSRP) from different nodes, and the reference signals from different nodes are usually orthogonal to each other [11, Ch. 22].

To improve the HO performance, optimizing $\mathrm{HO}$ parameters in different scenarios is one of the main topics in current research. Based on the scenarios and UE mobilities, several key performance indicators (KPIs) can be considered to evaluate the performance of $\mathrm{HO}$ algorithms [12], [13], [14]. In this paper, as our target is the VUE with moderate to high speed, we adopt the KPIs and methodologies used in [12], i.e., the observed OP, and the handover ping-pong ratio to optimally choose the value of HO offset and TTT. In a practical mobile communication system, on the one hand, sufficiently large values of $\mathrm{HO}$ offset and TTT are required to reduce the number of ping-pong HOs. On the other hand, too large values of HO offset and TTT will result in radio link failures which increase the observed $\mathrm{OP}$ of the system. Thus, a desired tradeoff between the two aforementioned KPIs need to be considered when choosing the values of HO offset and TTT.

\section{B. HO parameter optimizations}

Let $M$ and $T$ denote the values of HO offset and TTT, respectively. In our setup, if the received signal power of BSto-VUE direct transmission is $M$ times lower than the signal power of the FRN-to-VUE or MRN-to-VUE transmission for a length of $T$ seconds, the VUE will be handed over from the BS to the FRN or the MRN, and vice versa. If a UE is handed over to a new node, and is handed back to the source node within a critical time $T_{\mathrm{c}}$, we consider such a $\mathrm{HO}$ is a ping-pong $\mathrm{HO}$ [14]. For a given VUE at a given position, and during an observation time period of $T_{\mathrm{o}}$, where $T_{\mathrm{o}} \gg T_{\mathrm{c}}$, the ping-pong $\mathrm{HO}$ ratio $R_{\mathrm{p}}$ is defined by the ratio between the number of ping-pong HOs $N_{\mathrm{p}}$ and the total number of HOs $N_{\mathrm{t}}$, given as

$$
R_{\mathrm{p}}\left(x_{1}, M, T\right)=\left\{\begin{array}{ll}
\frac{N_{\mathrm{p}}\left(x_{1}, M, T\right)}{N_{\mathrm{t}}\left(x_{1}, M, T\right)}, & N_{\mathrm{t}}\left(x_{1}, M, T\right) \neq 0 \\
0, & N_{\mathrm{t}}\left(x_{1}, M, T\right)=0
\end{array} .\right.
$$

In theory, due to shadowing, small scale fading or even the weather, regardless the position of a UE in its serving cell, there is always a probability that a UE can be handed over to a neighboring BS; however, in practical systems, the probably that HO happens when a UE is near its serving BS can hardly be observed.

As indicated in Section IV-A, there is a trade off between $P_{\text {out }}$ and $R_{\mathrm{p}}$, i.e., they cannot be minimized at the same time. Thus, a common approach to such multi-objective optimization problems is to focus on one of the objectives while constraining the other objective to a certain predefined target. We follow the approaches in [12], and formulate the optimization problem as

$$
\begin{array}{cl}
\underset{(M, T)}{\operatorname{minimize}} & \bar{P}_{\text {out }}\left(M, T, \gamma_{\mathrm{th}}\right) \\
\text { subject to } & \bar{R}_{\mathrm{p}}\left(M, T, \gamma_{\mathrm{th}}\right)<R_{\mathrm{t}},
\end{array}
$$

where $R_{\mathrm{t}}$ is a given average ping-pong target rate. $\bar{P}_{\text {out }}$ and $\bar{R}_{\mathrm{p}}$ are the average OP and ping-pong rate ${ }^{1}$, respectively, with a given VUE distribution. Let $f_{x_{1}}\left(x_{1}\right)$ denote the pdf of the UE position distribution, and for a given $M$ and $T$ we have

$$
\begin{gathered}
\bar{P}_{\text {out }}\left(M, T, \gamma_{\mathrm{th}}\right)=\int_{0}^{\infty} P_{\text {out }}\left(x_{1}, M, T, \gamma_{\mathrm{th}}\right) f_{x_{1}}\left(x_{1}\right) d x_{1}, \\
\bar{R}_{\mathrm{p}}\left(M, T, \gamma_{\mathrm{th}}\right)=\int_{0}^{\infty} R_{\mathrm{p}}\left(x_{1}, M, T, \gamma_{\mathrm{th}}\right) f_{x_{1}}\left(x_{1}\right) d x_{1} .
\end{gathered}
$$

In this study, since we consider VUEs moving along a road (see Fig. 1), it is reasonable to assume a uniform position distribution of the VUE. For the MRN assisted transmission, as we assume no fading for the access link, for a given $M$ and $T$, the average OP $\bar{P}_{\text {out }_{M}}$ can be obtained by using (3). The threshold $\gamma_{\text {th }}$ in (8) needs to be determined based on whether

\footnotetext{
${ }^{1}$ Due to HO parameters are cell-specific rather than UE-specific, the OP and ping-pong ratio need to be averaged over certain UE distributions.
} 
Table I

Optimal $M, T$, AND CORRESPONDING $\bar{P}_{\text {out }_{M}}\left(M, T, \gamma_{\text {th }}\right)$ AGAinst DIFFERENT VPL WITH TARGET $\bar{R}_{\mathrm{p}}(M, T)<0.2$ FROM MRN

\begin{tabular}{|c|c|c|c|}
\hline VPL $[\mathrm{dB}]$ & $M[\mathrm{~dB}]$ & $T[\mathrm{~ms}]$ & $\bar{P}_{\text {out }_{\mathrm{M}}}\left(M, T, \gamma_{\mathrm{th}}\right)$ \\
\hline \hline 0 & 3 & 320 & 0.0159 \\
\hline 10 & -2 & 40 & $1.9386 \mathrm{e}-3$ \\
\hline 30 & 2 & 40 & $1.9206 \mathrm{e}-3$ \\
\hline
\end{tabular}

the VUE is served by the BS or the RN (see eq. ( 10) as an example). The FRN case is discussed in Section IV-C.

We remark that the choices $M$ and $T$ highly depend on the VUE velocity. To monitor the received power, the receiver needs to sample the received signals from different nodes. The sampled received power during the given time $T$ can be regarded as time correlated joint random variables, due to the randomness of wireless channels, and the time correlation depends on the VUE speed. To calculate the exact expression of (6) and (9), the joint cumulative distribution function (cdf) of the power samples is needed. The joint pdf of time correlated Rayleigh distributed random variables is a series of products of univariate gamma pdfs [15]; however, the closed form expression of the cdf is unknown. If the number of observations is big, it is even difficult to calculate the cdf numerically. In some cases, e.g., VUEs with high velocity, the sampled received power at each observation time can be regarded as independent [12], [13]. Then approximations of $\bar{P}_{\text {out }}\left(M, T, \gamma_{\mathrm{th}_{\mathrm{R}}}\right)$ and $\bar{R}_{\mathrm{p}}(M, T)$ can be obtained, and low complexity algorithms can be developed accordingly to optimize the choice of $M$ and $T$. But for low to moderate speed of the VUEs, such approximations are not accurate enough. In this paper, we are aiming at performance evaluations, and thereby we resort to extensive system simulations by using exhaustive search to obtain the optimal values of $M$ and $T$.

\section{The optimal FRN position problem}

In order to benefit from the FRN and to achieve a fair comparison between different schemes, the FRN should be deployed in an optimal position that minimize the average endto-end OP at the VUE. Neglecting the ping-pong effect, if the VUE is handed over from the BS to the FRN at the position $l$ and handed back from the FRN to the BS at position $l^{\prime}$, we have

$$
\begin{aligned}
\bar{P}_{\text {out }_{\mathrm{F}}} & \left(M, T, d, \gamma_{\mathrm{th}}\right) \\
& =\mathbb{E}_{x}\left[P_{\text {out }_{\mathrm{F}}}\left(M, T, d, x_{1}, \gamma_{\mathrm{th}}\right)\right] \\
& =\int_{0}^{l} P_{\text {out }_{\mathrm{D}}}\left(M, T, x_{1}, \gamma_{\mathrm{th}_{\mathrm{D}}}\right) f_{x_{1}}\left(x_{1}\right) d x_{1} \\
& +\int_{l}^{l^{\prime}} P_{\text {out }_{\mathrm{R}}}\left(M, T, x_{1}, \gamma_{\mathrm{th}_{\mathrm{R}}}\right) f_{x_{1}}\left(x_{1}\right) d x_{1} \\
& +\int_{l^{\prime}}^{D} P_{\text {out }_{\mathrm{D}}}\left(M, T, x_{1}, \gamma_{\mathrm{th}_{\mathrm{D}}}\right) f_{x_{1}}\left(x_{1}\right) d x_{1} .
\end{aligned}
$$

Thus the optimal FRN position is determined as

$$
\bar{d}_{\text {opt }}=\arg \min _{d_{\text {break }}<d \leq D} \bar{P}_{\text {out }_{\mathrm{F}}}\left(M, T, d, \gamma_{\text {th }}\right)
$$

subject to

$$
\bar{R}_{\mathrm{p}}(M, T)<R_{\mathrm{t}} \text {. }
$$

Table II

OptimAL $M, T, \bar{d}_{\text {opt AND CORRESPONDING }} \bar{P}_{\text {out }_{\mathrm{F}}}\left(d, \gamma_{\text {th }}\right)$ AGAINST DIFFERENT VPL WITH TARGET $\bar{R}_{\mathrm{p}}(M, T)<0.2$ FOR FRN

\begin{tabular}{|c|c|c|c|c|}
\hline $\begin{array}{c}\text { VPL } \\
{[\mathrm{dB}]}\end{array}$ & $\begin{array}{c}M \\
{[\mathrm{~dB}]}\end{array}$ & $\begin{array}{c}T \\
{[\mathrm{~ms}]}\end{array}$ & $\begin{array}{c}\bar{d}_{\mathrm{opt}} \\
{[\mathrm{m}]}\end{array}$ & $\bar{P}_{\mathrm{out}_{\mathrm{F}}}\left(d, \gamma_{\mathrm{th}}\right)$ \\
\hline \hline 0 & 3 & 40 & 805 & $9.6516 \mathrm{e}-04$ \\
\hline 10 & 3 & 40 & 825 & $1.5686 \mathrm{e}-3$ \\
\hline 30 & -2 & 40 & 805 & $5.4094 \mathrm{e}-3$ \\
\hline
\end{tabular}

Problem (11) is tackled by system level evaluations, the same as for (7), and the parameters used for the evaluations are given in Section VI. The optimal FRN poisons and corresponding $M, T$, and $\bar{P}_{\text {out }_{\mathrm{F}}}\left(d, \gamma_{\mathrm{th}}\right)$ are given in Table II. As we can see, the FRN should be deployed near to the cell edge in order to minimize the average OP.

\section{Propagation Models}

The propagation environments of the considered schemes are identified in [7]. BS-to-VUE direct transmission and its interference are considered to be non-line-of-sight (NLOS). For FRN assisted transmission, the backhaul link, i.e, BS to FRN, is considered to be line-of-sight (LOS). This is motivated by proper site planning, in which case the probability for the FRN backhaul link to have a LOS connection is relatively high. The interference of the FRN backhaul link, i.e., BS2 to FRN1, is modeled as NLOS, since the FRN is far away from the interfering BS and LOS probability in this case is very low (see [16, Table A.2.1.1.2-3]). Such a setup is the best scenario an FRN can experience in practice. Regarding the MRN assisted transmission, the pathloss of the backhaul link and its interference are modeled in the same way as the direct transmission.

For the access link, since the distance between the MRN and the VUE is short (up to around 5 meters), and there is almost always a LOS link, a constant power loss $G$ is assumed, which includes the effect of pathloss, shadowing and small scale fading. As for the interference between the access link of two MRNs, i.e., MRN2 to UE1, a LOS propagation environment is assumed.

However, in [7], the OP performance study only considers the effect of pathloss and small scale fading in a system without $\mathrm{HO}$ ability. In order to better understand the performance of the considered transmission schemes, in this work, we also consider the effect of shadowing based on well known channel models, which is discussed next. The pathloss and small scale fading models, and the choice of $G$ are aligned with [7], which are summarized in later parts of this section.

\section{A. Shadowing}

Usually, the average received signal power at the RX varies randomly at a given distance from the TX due to the blockage from objects in the propagation path, and this effect is usually called shadowing. Shadowing causes additional power attenuation to the pathloss at the receiver. One of the most common models for this power attenuation is log-normal shadowing [17, Ch. 2]. The log-normal distribution is parametrized by the $\log$ mean $\mu_{\mathrm{dB}}$ and standard deviation $\sigma_{\mathrm{dB}}$, and the probability 
Table III

SUMMARY OF THE SHADOWING STANDARD DEVIATIONS

\begin{tabular}{|c|c|c|}
\hline Scenario & $\begin{array}{c}\text { Standard } \\
\text { Deviation } \\
\sigma_{\mathrm{dB}}[\mathrm{dB}]\end{array}$ & Channel Model \\
\hline Direct transmission and its interference (BS1-UE1 and BS2-UE1) & \multirow{2}{*}{6} & \multirow{2}{*}{ 3GPP Urban Macro } \\
\hline $\begin{array}{l}\text { MRN backhaul link and its interference (BS1-MRN1 and } \\
\text { BS2-MRN1) }\end{array}$ & & \\
\hline FRN backhaul link (BS1-FRN1) & 3.4 & $\begin{array}{l}\text { WINNER II B5a (LOS stationary feeder: } \\
\text { rooftop to rooftop) }\end{array}$ \\
\hline Interference to the backhaul link of FRN (BS2-FRN1) & 6 & 3GPP NLOS Macro BS to outdoor FRN \\
\hline 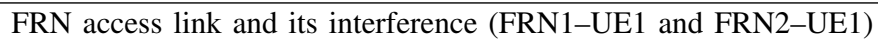 & 10 & 3GPP NLOS outdoor FRN to VUE \\
\hline Interference to the access link of MRN (MRN2-UE1) & 3 & $\begin{array}{l}\text { WINNER II B5b (LOS stationary feeder: } \\
\text { street level to street level) }\end{array}$ \\
\hline
\end{tabular}

density function (pdf) can be found in [17, Ch. 2]. The value of $\mu_{\mathrm{dB}}$ equals the empirical pathloss between the TX and RX, and a value of $3-10 \mathrm{~dB}$ is observed for the standard deviation $\sigma_{\mathrm{dB}}$ in different scenarios.

In most cases of this study, we set the values of the standard deviation $\sigma_{\mathrm{dB}}$ according to the 3GPP channel models, given in [16, Table A.2.1.1.2-3] and [16, Table B.1.2.1-1]. However, for cases such as the LOS BS to FRN link and the interference between two MRNs, which are not studied in 3GPP, we refer to the WINNER II channel models [18]. It is worth mentioning that even though in the LOS propagation, i.e., BS1 to FRN1 and MRN2 to UE1, the probability that the direct path is shadowed is very low, but the reflective paths can still be shadowed. Thus, a lower shadowing standard deviation is observed in the LOS propagation environment compared to the NLOS case. The different values of the standard deviations and the corresponding considered channel model are summarized in Table III.

\section{B. Pathloss models}

The pathloss $L(y)$ is often determined by measurements [8, Ch. 4], and can usually be modeled in logarithmic scale as

$$
L(y)[\mathrm{dB}]=A \log _{10}(y)+B,
$$

where $A$ is the pathloss exponent, $B$ is the pathloss constant and $y$ is the distance between TX and RX. ${ }^{2}$ It is worth mentioning that pathloss models are usually valid when the distance between a TX and an RX is greater than a certain value, also known as the break point $[8, \mathrm{Ch}$. 4]. As the detailed pathloss modeling is out of the scope of this study, for simplicity, within the break point, we conservatively assume that the pathloss is constant and equals the pathloss at the break point distance, i.e., $L(y)=L\left(y_{\text {break }}\right), y \leq y_{\text {break }}$.

In our study, a carrier frequency of $2.0 \mathrm{GHz}$ is considered and we follow the recommendation settings in [16] for BS-toVUE direct transmission and FRN assisted transmission. Regarding the MRN assisted transmission, as mentioned above, a constant power loss $G$ which includes the effect of pathloss, shadowing and small scale fading is considered for its access

\footnotetext{
${ }^{2}$ The distance in this study is measured in kilometers. The values of $A$ and $B$ given in Table IV will be different when other units are used.
}

link. In this study, we set $G=-84 \mathrm{~dB}$ which approaches the measurement lower bound shown in [19] under the presence of shadowing and small scale fading. As for the interference between the access link of two MRNs, i.e., MRN2 to UE1, we use the LOS COST 231-Walfish-Ikegami pathloss model [20, Ch. 7]. The values of $A$ and $B$ for different propagation scenarios are summarized in Table IV.

\section{Small scale fading}

Small scale fading is referred to rapid amplitude variation at the RX caused by multi-path propagations [21, Ch. 3.2.2]. Depending on the environments, there are several models available to describe the effect of small scale fading. In this study, the amplitude of the channel coefficients are considered to be Rayleigh distributed in a NLOS propagation environment and a Rician distribution is considered in a LOS propagation environment [21, Ch. 3.2.2]. The Rician distribution is characterized by its $K$ factor. Based on the studies of stationary feeder links in typical urban scenarios [18, pp. 17-18], we consider $K_{\mathrm{F}_{\mathrm{bk}}}=10$ for the FRN backhaul in this study. For the street level LOS propagation, the $K$ factor depends on the distance between the TX and the RX. In our study, we adopt the model given in [18, pp. 73] and the $K$ factors for the interference to access link of the serving MRN, i.e., MRN2 to UE1, is given as follows.

$$
K_{\mathrm{M}_{\mathrm{I}_{\mathrm{ac}}}}=\left\{\begin{array}{lr}
10 & L_{\mathrm{M}_{\mathrm{I}}}\left(y_{\mathrm{I}}\right)<85 \mathrm{~dB} \\
2 & 85 \mathrm{~dB} \leq L_{\mathrm{M}_{\mathrm{I}}}\left(y_{\mathrm{I}}\right)<110 \mathrm{~dB} \\
1 & L_{\mathrm{M}_{\mathrm{I}}}\left(y_{\mathrm{I}}\right) \geq 110 \mathrm{~dB}
\end{array} .\right.
$$

\section{Vi. Performance Evaluation}

In this section, we evaluate and compare VUE's OP of the considered schemes, i.e., direct transmission and FRN and MRN assisted transmission. The employed evaluation parameters are based on [16], [22] and summarized in Table V. The MRNs are placed on top of the vehicle and assumed to eliminate VPL. The optimal HO parameters and FRN position that minimize the overall average OP are obtained according to the discussion in Section IV. When searching for the optimal HO parameters, the $\mathrm{HO}$ offset values vary between $-3 \mathrm{~dB}$ and $10 \mathrm{~dB}$ with steps of $1 \mathrm{~dB}$, and the values of TTT are enumerated from the values specified by the 3GPP LTE 
Table IV

SUMMARY OF THE PATHLOSS MODELS

\begin{tabular}{|c|c|c|c|c|}
\hline Scenario & LOS / NLOS & $A$ & $B$ & Break Point $[\mathrm{km}]$ \\
\hline Direct transmission and its interference (BS1-UE1 and BS2-UE1) & \multirow{2}{*}{ NLOS } & \multirow{2}{*}{42.8} & \multirow{2}{*}{131.1} & \multirow{2}{*}{0.035} \\
\hline MRN backhaul link and its interference (BS1-MRN1 and BS2-MRN1) & & & & \\
\hline FRN backhaul link (BS1-FRN1) & LOS & 23.5 & 100.7 & 0.035 \\
\hline Interference to the backhaul link of FRN (BS2-FRN1) & NLOS & 36.3 & 125.2 & 0.035 \\
\hline FRN access link and its interference (FRN1-UE1 and FRN2-UE1) & NLOS & 37.5 & 145.4 & 0.035 \\
\hline Interference to the access link of MRN (MRN2-UE1) & LOS & 26 & 108.6 & 0.02 \\
\hline Access link of MRN (MRN1-UE1) & LOS & \multicolumn{3}{|c|}{ Constant power loss $G=-84 \mathrm{~dB}$} \\
\hline
\end{tabular}

Table V

SimUlation PARAMETERS

\begin{tabular}{|c|c|}
\hline Parameter & Value \\
\hline \hline Inter-site Distance between BS1 and BS2 & $1732 \mathrm{~meters}$ \\
\hline Average BS transmit power & $46 \mathrm{dBm}$ \\
\hline Average FRN transmit power & $30 \mathrm{dBm}$ \\
\hline Average MRN transmit power & $20 \mathrm{dBm}$ \\
\hline Carrier Frequency & $2.0 \mathrm{GHz}$ \\
\hline System Bandwidth & $10 \mathrm{MHz}$ \\
\hline $\begin{array}{c}\text { Receiver noise figure for both the RN } \\
\text { and the VUE }\end{array}$ & $9 \mathrm{~dB}$ \\
\hline $\begin{array}{c}\text { Normalized Minimum Required Rate } R \\
\text { at the VUE }\end{array}$ & $1 \mathrm{bit} / \mathrm{s} / \mathrm{Hz}$ \\
\hline VUE Velocity & $120 \mathrm{~km} / \mathrm{h}$ \\
\hline HO critical time $T_{\mathrm{c}}$ & $5 \mathrm{~seconds}$ \\
\hline HO performance observation time $T_{\mathrm{o}}$ & $200 \mathrm{~seconds}$ \\
\hline Shadowing de-correlation distance & $50 \mathrm{~meters}$ \\
\hline
\end{tabular}

standard [22, Section 6.3.5]. These values are $\left(\begin{array}{lll}0 & 0.04 & 0.064\end{array}\right.$ $\begin{array}{lllllllllllll}0.08 & 0.1 & 0.128 & 0.16 & 0.256 & 0.32 & 0.48 & 0.512 & 0.64 & 1.024 & 1.280\end{array}$ 2.560 ) in seconds. The HO decisions are made independently in both of the primary and interfering cells based on the measurement of RSRP, and UE2 is dropped uniformly in the interfering cell.

We move UE1 from its serving BS to the cell edge and plot its OP for the considered setups. As shown from the results (Figs. 2-4), the VUE is served by the BS when it is near to it, and handed over to the FRN or MRN afterwards. Regarding the MRN assisted transmission, from both Table I and Figs. 2-4 we can see that it gives a lower OP at the VUE when the VPL is moderate to high. This is not only because that the MRN can eliminate VPL but also it can dampen the CCI from the interfering cell. As shown in Fig. 3, the OP at the VUE of the MRN assisted transmission begin to approach the direct transmission when the VPL is $10 \mathrm{~dB}$ with a small difference due to the half-duplex loss. But the MRN gradually outperforms the direct transmission when the VPL increases. This can be clearly observed from Fig. 4. After performing the $\mathrm{HO}$, the OP at the VUE is significantly lower for the MRN assisted transmission compared to other two schemes.

From the evaluation results, we can see that the traditional RSRP based HO can be further improved for the MRN assisted transmission. On the one hand, it is clear in Fig. 2 that when there is no VPL even if the signal from the MRN is stronger

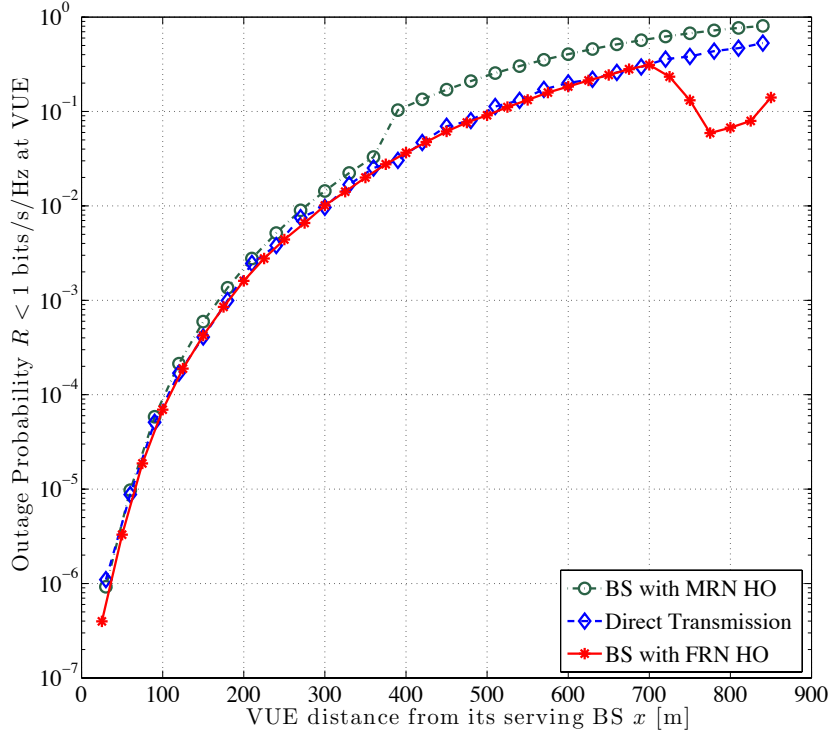

Figure 2. OP performance when VPL $=0 \mathrm{~dB}$

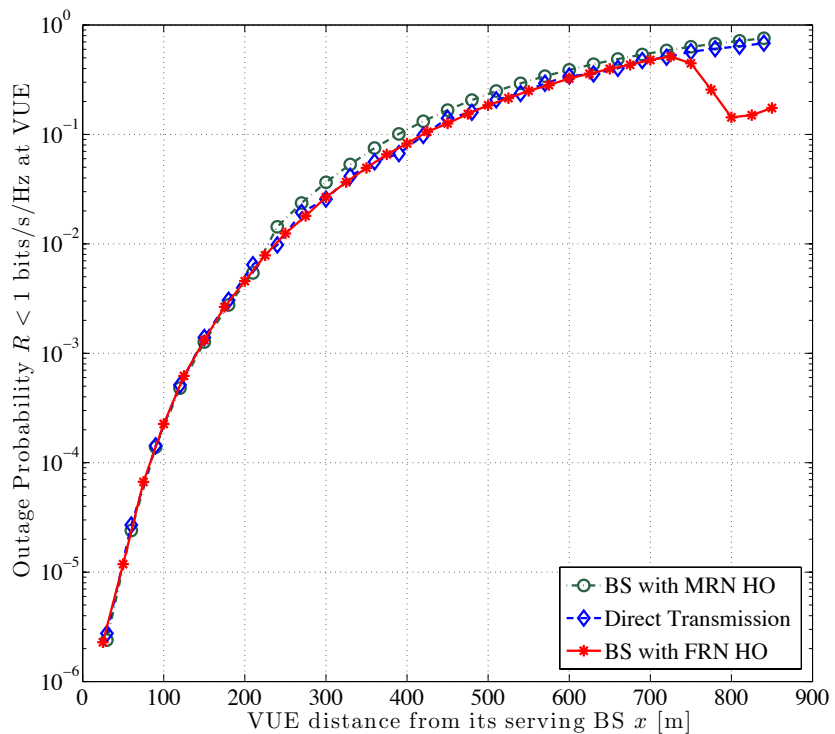

Figure 3. OP performance when $\mathrm{VPL}=10 \mathrm{~dB}$

but the OP at the VUE will not be lowered due to the halfduplex loss. Similar but less obvious behaviors can also be seen when VPL is $10 \mathrm{~dB}$. On the other hand, even if the VPL is high (see Fig. 4), due to the high BS transmit power and the HO offset, the VUE will not be handed over to the MRN until it is around 100 meters away from the BS. The OP at the VUE could be further lowered at high VPL, if the VUE is served by the MRN directed from the very beginning. As discussed 


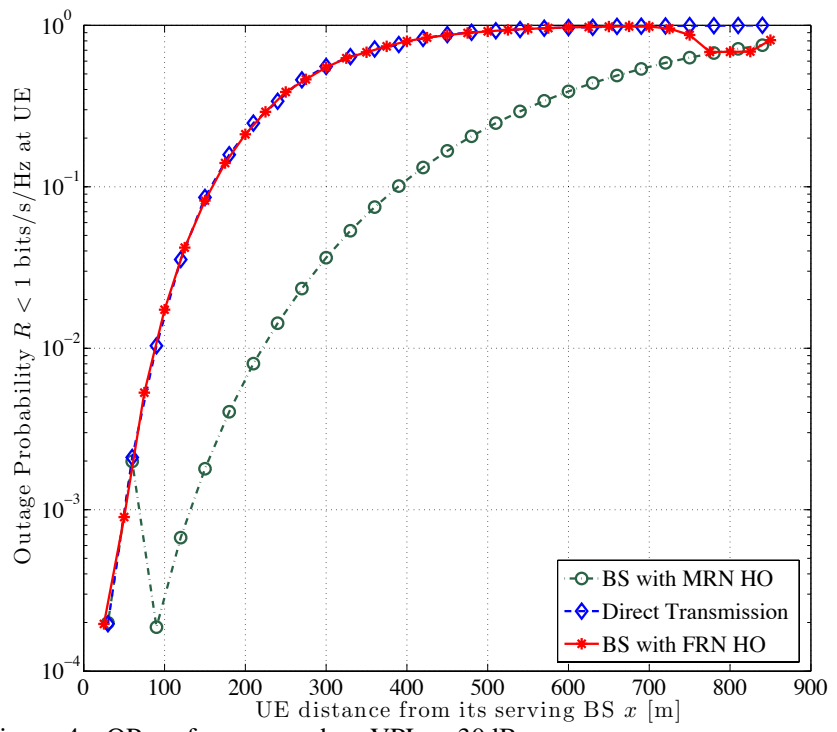

Figure 4. OP performance when $\mathrm{VPL}=30 \mathrm{~dB}$

in Section IV, in current systems, the HO parameters are cellspecified rather than UE-specified. In the future system, if the HO parameters can be adjusted based on each of the MRNs, better performance can be expected.

Furthermore, as expected in all the cases, since the FRN can better compensate the pathlosses, it serves the VUE more efficiently when the VUE is handed over to it, despite the half-duplex loss. Nevertheless, on average, it still cannot outperform the MRN assisted transmission when the VPL is moderate to high. But since the FRN is designed for coverage extension, such a behavior is expected. It is worth mentioning that, as described in Section V, we model the backhaul link of the FRN assisted transmission as LOS but its interference is modeled as NLOS. This is the best case one can expect for the FRN assisted transmission, but even with these assumptions, the contribution of FRN to the VUE is very limited at moderate to high VPLs. Another thing that is worth mentioning is that the MRN is assumed to operate at a much lower transmit power than the FRN, but it serves VUEs better than the FRN assisted transmission on average. Thus from an energy efficiency point of view, MRN is also a better choice for serving VUEs.

\section{CONCLUSIONS}

In this paper, we compare the end-to-end outage performance at a vehicular user of single-hop direct transmission (baseline case), and dual-hop transmission via a moving relay node as well as a fixed relay node. We targeted practical scenarios by employing different channel models of the different links, and involved the effect of handover between a base station and relay nodes. We show that in the case of moderate to high vehicular penetration loss, a moving relay node deployed on top of public transportation vehicles can bring significant enhancement to the quality-of-service the VUE compared to the direct and fix relay assisted transmission. Thus, the use of moving relay nodes have a very good potential to boost performance of future mobile communication systems.

\section{ACKNOWLEDGMENT}

Part of this work has been performed in the framework of the FP7 project ICT-317669 METIS, which is partly funded by the European Union. The authors would like to acknowledge the contributions of their colleagues in METIS, although the views expressed are those of the authors and do not necessarily represent the project. This work is also supported by the Swedish Research Council VR under the project 621-20094555 Dynamic Multipoint Wireless Transmission.

\section{REFERENCES}

[1] P. Popovski, V. Braun, H.-P. Mayer, P. Fertl, et al., "ICT-317669-METIS/ D1.1 Scenarios, requirements and KPIs for 5G mobile and wireless system," tech. rep., 2013. [Online]: https://www.metis2020.com/.

[2] 3GPP TR 36.836, "Technical Specification Group Radio Access Network; Mobile Relay for Evolved Universal Terrestrial Radio Access (EUTRA)," tech. rep. [Online] http://www.3gpp.org/ accessed 2013-07-05.

[3] E. Tanghe, W. Joseph, L. Verloock, and L. Martens, "Evaluation of vehicle penetration loss at wireless communication frequencies," IEEE Trans. Veh. Technol., vol. 57, pp. 2036-2041, Jul. 2008.

[4] W. Li, C. Zhang, X. Duan, S. Jia, et al., "Performance evaluation and analysis on group mobility of mobile relay for LTE-Advanced system," in Proc. IEEE VTC 2012-Fall.

[5] V. V. Phan, K. Horneman, L. Yu, and J. Vihriala, "Providing enhanced cellular coverage in public transportation with smart relay systems," in Proc. IEEE VNC, 2010.

[6] Y. Sui, A. Papadogiannis, and T. Svensson, "The potential of moving relays-a performance analysis," in Proc. IEEE VTC 2012-Spring.

[7] Y. Sui, A. Papadogiannis, W. Yang, and T. Svensson, "Performance comparison of fixed and moving relays under co-channel interference," in Proc. IEEE GLOBECOM Workshops, 2012. to appear.

[8] A. F. Molisch, Wireless Communications. West Sussex, UK: John Wiley \& Sons Ltd., 2nd edition ed., 2010.

[9] J. M. Romero-Jerez and A. J. Goldsmith, "Receive antenna array strategies in fading and interference: An outage probability comparison," IEEE Trans. Wireless Commun., 2008.

[10] P. Mogensen, W. Na, I. Kovacs, et al., "Lte capacity compared to the shannon bound," in Proc. IEEE VTC 2007-Spring, 2007.

[11] S. Sesia, I. Toufik, and M. Baker, LTE - The UMTS Long Term Evolution: From Theory to Practice. West Sussex, UK: John Wiley \& Sons Ltd. 2nd edition ed., 2011.

[12] Z. Ren, P. Fertl, Q. Liao, et al., "Street-specific handover optimization for vehicular terminals in future cellular networks," in Proc. IEEE VTC 2013-Spring Workshop, 2013.

[13] Q. Liao, F. Penna, S. Stanczak, et al., "Context-aware handover optimization for relay-aided vehicular terminals," in Proc. IEEE IEEE 14th SPAWC Workshop, 2013.

[14] T. Jansen, I. Balan, J. Turk, et al., "Handover parameter optimization in lte self-organizing networks," in Proc. IEEE VTC 2010-Fall, 2010.

[15] R. K. Mallik, "On multivariate rayleigh and exponential distributions," IEEE Trans. Inf. Theory, vol. 49, pp. 1499-1515, June 2003.

[16] 3GPP TR 36.814, "Evolved Universal Terrestrial Radio Access (EUTRA); Further advancements for E-UTRA physical layer aspects," tech. rep. [Online] http://www.3gpp.org/ accessed 2013-09-26.

[17] T. Rappaport, Wireless Communications: Principles and Practice. New Jersey, USA: Prentice Hall, 2nd edition ed., 2002.

[18] P. Kyösti, J. Meinilä, L. Hentilä, X. Zhao, et al., "WINNER II Channel Models Part II, Radio channel measurement and analysis results," tech. rep., IST-4-027756 WINNER II, 2007. [Online] http://www.istwinner.org/deliverables.html accessed 2012-06-12.

[19] H. Masui, T. Kobayashi, and M. Akaike, "Microwave path-loss modeling in urban line-of-sight environments," IEEE J. Sel. Areas Commun., vol. 20, pp. 1151-1155, Aug 2002.

[20] A. F. Molisch, Supplementary Material for 2nd Edition of Wireless Communications. West Sussex, UK: John Wiley \& Sons Ltd., 2011. [Online] Available: http://www.wiley.com/ accessed 2012-03-28

[21] A. Goldsmith, Wireless Communications. New York, USA: Cambridge University Press, 2005.

[22] 3GPP TS 36.331, "Evolved Universal Terrestrial Radio Access (EUTRA); Radio Resource Control (RRC); Protocol specification," tech. rep., 2013. [Online] http://www.3gpp.org/ accessed 2013-09-26. 